\title{
Stage IB: To give (chemo), or not to give? That is the question
}

\author{
Joseph B. Shrager, MD
}

\author{
From the Division of Thoracic Surgery, Stanford University School of Medicine and Stanford Cancer Institute, \\ Stanford, Calif. \\ Disclosures: Author has nothing to disclose with regard to commercial support. \\ Received for publication Oct 6, 2017; accepted for publication Oct 9, 2017; available ahead of print Nov 8, 2017. \\ Address for reprints: Joseph B. Shrager, MD, 300 Pasteur Dr, Falk Building, Stanford, CA 94305-5407 (E-mail: \\ shrager@stanford.edu). \\ J Thorac Cardiovasc Surg 2018;155:1206 \\ $0022-5223 / \$ 36.00$ \\ Copyright $($ c 2017 by The American Association for Thoracic Surgery \\ https://doi.org/10.1016/j.jtcvs.2017.10.016
}

Patients with stage IB non-small cell lung carcinoma sit directly at the transition point between those for whom we routinely recommend chemotherapy after resection and those for whom we routinely do not. The answer to the question of whether patients who have undergone resection for stage IB disease should receive adjuvant chemotherapy has varied during the past several years, from "always," to "never," to "yes, if larger than $4 \mathrm{~cm}$," to more complex formulations that take into account individual tumor biology.

In this issue of the Journal, our colleagues from the Shanghai Chest Hospital ${ }^{1}$ have made a substantial contribution to this discussion by examining the influence of one measure of the biologic aggressiveness of stage IB adenocarcinomas on survival after resection and on the impact of adjuvant chemotherapy. They examined a large cohort of patients with stage IB adenocarcinoma (eighth edition; 3-4 cm N0 tumors) with sufficiently long median follow-up (47 months) to draw firm conclusions. They used propensity score modeling in the analysis to determine whether adjuvant chemotherapy was beneficial.

They have focused their attention on the influence of the extent of micropapillary or solid histologic type within stage IB adenocarcinomas. These subtypes, defined in the adenocarcinoma histologic classification proffered in 2011, have previously been established as conferring worse prognosis, and this study confirms that finding. More importantly, this study provides stronger data than previous studies that administering adjuvant chemotherapy in patient with histologically higher risk tumors actually improves outcomes. In wellmatched groups of patients with tumors bearing a predominant solid or micropapillary pattern, the cumulative incidence of lung cancer-specific death was dramatically lower in those who received adjuvant chemotherapy than in those who did not (hazard ratio, 0.46; confidence interval, 0.22-0.93). ${ }^{1}$

These results are of major interest. Focusing on tumor biology in this fashion is likely to provide a better means than crude measures such as size of distinguishing between IB tumors that should and should not be considered for adjuvant chemotherapy. Given the recently appreciated importance of micropapillary histologic type, this is a smart place to start (although in the future, genomic markers are likely to prove even more important). These results cannot,

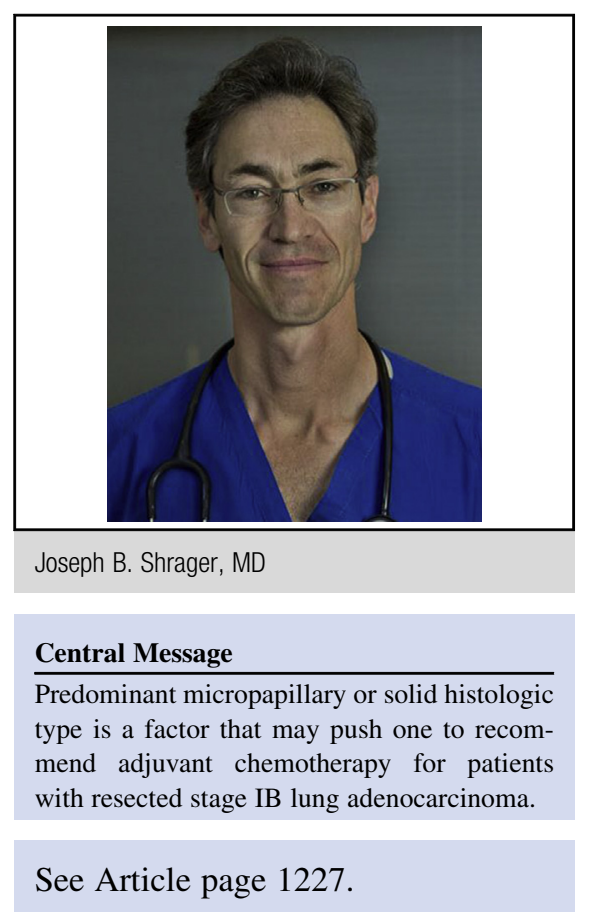

however, be considered conclusive. There likely remain potential confounders that have not been accounted for by their propensity scoring. Additionally, a Chinese cohort of lung adenocarcinomas will differ markedly from one in North America or Europe-with, for example, far more tumors in nonsmokers $(67 \%$ in this study) and bearing epidermal growth factor receptor mutations. These tumors behave very differently than the typical adenocarcinomas in countries with predominant European-ancestry and African-ancestry populations. It is unfortunately very likely that conclusions about this disease drawn from patients of one ethnicity will not apply to those of others.

So, as is nearly always the case, one must conclude that additional prospective, randomized studies would be required to sort out truly which patients with stage IB adenocarcinomas should receive adjuvant chemotherapy after resection. Only in this case, sorting out the question is even more complicated than usual-it would require separate prospective randomized trials in Asian and non-Asian populations! In the meantime, I do think that data from well-controlled retrospective studies such as this one can be brought to bear in the decision-making process for individual patients.

\section{Reference}

1. Qian F, Yang W, Wang R, Xu J, Wang S, Zhang Y, et al. Prognostic significance and adjuvant chemotherapy survival benefits of a solid or micropapillary pattern in patients with resected stage IB lung adenocarcinoma. J Thorac Cardiovasc Surg. 2018;155:1227-35. 